\title{
Reliability and responsiveness of a graduated tuning fork in immune mediated polyneuropathies
}

\author{
I S J Merkies, P I M Schmitz, F G A van der Meché, P A van Doorn, for the Inflammatory
} Neuropathy Cause and Treatment (INCAT) Group

\begin{abstract}
The interobserver and intraobserver reliability of the Rydel-Seiffer (RS) graduated tuning fork was evaluated in 113 patients with a clinically stable immune mediated polyneuropathy (83 patients who had had Guillain-Barré syndrome (GBS) in the past, 22 with a chronic inflammatory demyelinating polyneuropathy (CIDP), and eight with a polyneuropathy associated with a gammopathy of undetermined significance). Additionally, the responsiveness of this instrument was serially investigated in 20 patients with recently diagnosed GBS or CIDP and changing clinical conditions. The measures were done in triplicate at eight different locations in the limbs and the values were compared with the recently published vibration threshold reference values. Good interobserver and intraobserver agreements (quadratic weighted $\kappa=0.67-$ 0.98 ) and high responsiveness values (standardised response mean scores $>0.8$ ) were demonstrated for the RS tuning fork. These results provide, in addition to literature findings, further evidence for incorporation of this easily applicable instrument in routine neurological examination.

(F Neurol Neurosurg Psychiatry 2000;68:669-671)
\end{abstract}

Department of

Neurology, Daniel den

Hoed Cancer Centre,

University Hospital

Rotterdam/Erasmus

University, Dr

Molewaterplein 40,

3015 GD, Rotterdam,

The Netherlands

I S J Merkies

F G A van der Meché

P A van Doorn

Department of Statistics

P I M Schmitz

Correspondence to:

Dr I S J Merkies

email merkies@neur.azr.nl

Received 26 August 1999 and in revised form

14 December 1999

Accepted 5 January 2000
Keywords: graduated tuning fork; reliability; responsiveness; immune mediated polyneuropathy

Clinically useful vibration threshold reference values were recently published for the RydelSeiffer (RS; Martin, Tuttlingen, Germany) graduated tuning fork and its construct validity was also demonstrated (figure). ${ }^{1}$ In addition, we investigated the reliability and responsiveness of this fork in patients with immune mediated polyneuropathies. ${ }^{2}$ Patients with GuillainBarré syndrome (GBS), chronic inflammatory demyelinating polyneuropathy (CIDP), or a polyneuropathy associated with a monoclonal gammopathy of undetermined significance (MGUSP) were recruited, as it is was argued that these disorders represent parts of a continuum of neuromuscular dysfunction pattern. $^{3}$
Patients and methods

PATIENTS

One hundred and thirteen patients (83 with GBS, 22 with CIDP, and eight with MGUSP) with a stable neurological condition were recruited from the Rotterdam immune mediated polyneuropathy databank and the Dutch GBS study group (stable group). Nine patients with CIDP required interval treatment, ranging from weeks to months, with intravenous immunoglobulin (IVIg). With this therapy their clinical condition has been stable for more than 6 months. Twenty other patients recently diagnosed with GBS $(n=7)$ or CIDP $(n=13)$ were enrolled to investigate the responsiveness of the RS tuning fork (longitudinal group). All patients with GBS or CIDP met the research criteria for their illness. ${ }^{45}$ The diagnosis MGUSP was established after excluding all possible causes for the gammopathy and polyneuropathy. ${ }^{6}$

ASSESSMENT TOOL AND TEST PROCEDURE

The RS tuning fork is a graduated fork that determines the ability of subjects to discriminate various vibration intensities (figure). ${ }^{17} \mathrm{~A}$ triangle and an arbitrary scale imprinted on the weights (range 0 (minimum score) to 8 (maximum score)) of the arms allow assessment of vibration threshold. Once the arms are swinging, the fork vibrates at $64 \mathrm{~Hz}$ and the triangles on the weights appear double. The intersection of these two virtual triangles moves from 0 to 8 in an exponential way with decreasing vibration amplitude of the arms. ${ }^{1}$ The vibration extinction threshold is considered as the nearest value to the apparent point of intersection of the virtual triangles when the patient indicates that vibration was no longer perceived.

The fork was applied as perpendicular as possible with its arms swinging maximally. The patients lay at ease in a supine position. The vibration extinction threshold was calculated by averaging the readings of three repeated tests. The averaged values were compared with the recently reported vibration threshold normal values and graded as follows: normal (grade $=0$ ) or disturbed (grade $=1$ ) vibration sense at the dorsum distal interphalangeal joint of the index finger or hallux; abnormal sense at the ulnar styloid process or medial malleolus (grade $=2)$; at the medial humerus epicondyle 


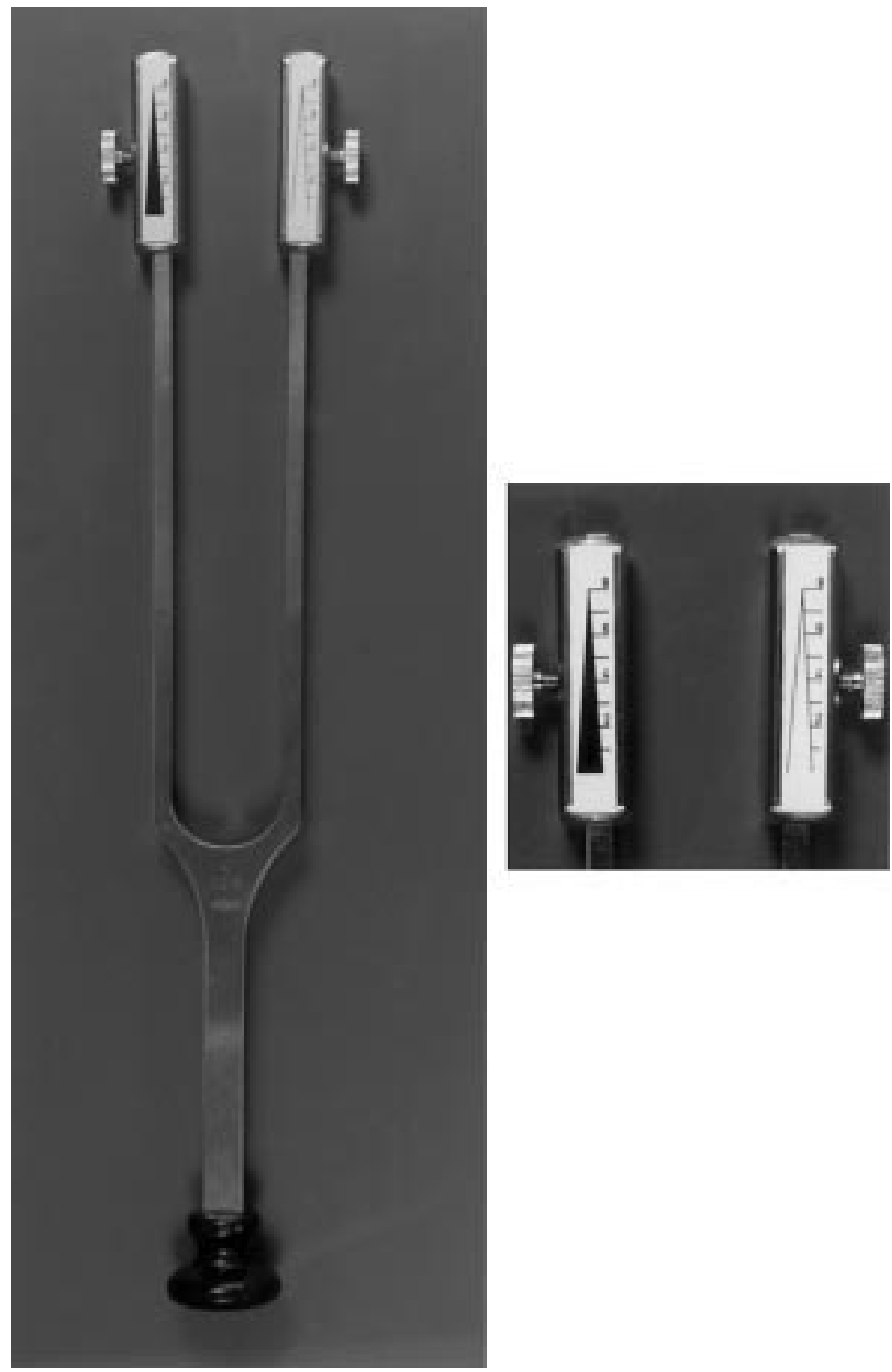

The Rydel-Seiffer (RS) tuning fork (Martin, Tuttlingen, Germany). Note the triangle and arbitrary scale from 0 (minimum score) to 8 (maximum score) imprinted on the weights of the two arms that allow quantitative vibration assessment. ${ }^{1}$

or patella (grade $=3$ ); at the acromioclavicular joint or anterior superior iliac spine $($ grade $=4){ }^{1}$ Examination took place from distal to proximal and only the most affected side and the highest extension of dysfunction were registered for the arms and legs separately. All measures were obtained in a quiet temperature controlled room at our outpatient clinic.

All participants gave informed consent before the study. For the reliability assessment of the fork, two neurologists and six residents in neurology formed 28 different pairs of examiners. Twenty seven ("variable") couples examined a total of 68 patients (two to three patients a couple). The remaining 45 patients were investigated by the "experienced" couple (couple No 1). This couple was formed to evaluate the effect of training and thus a possible increase in reliability when using the RS tuning fork more often. The stable patients were examined at two different occasions at our outpatient clinic. During the first visit the two members of an appointed pair performed their scores independently and consecutively (usually within 2 hours) (interobserver measures). Within 2-4 weeks, the patient returned for a second visit and only one investigator of the earlier assigned pair of researchers examined the patient again without having access to previous results (intraobserver measures). The assessments sequence at entry and the examination at the second visit were equally distributed among the members of an assigned couple. Overall, each member of a couple examined roughly the same number of patients as their partner.

For the responsiveness studies, all assessments were performed by the same clinician (ISJM) at study entry and 8-13 times in each patient during follow up. The study took place between March 1997 and July 1999.

\section{STATISTICS}

The interobserver/intraobserver reliability for the obtained vibration sense grades was determined using the weighted $\kappa$ statistic measures for the two investigators ("experienced" and "variable") groups. ${ }^{8}$ Responsiveness was investigated by calculating the standardised response mean (SRM) score for the total (arm (grade: $0-4)+$ leg (grade 0-4)) vibration grades (range $0-8$ ) at various arbitrarily chosen occasions during follow up (weeks 12, 26, 40, and 52). ${ }^{9}$ The SRM is equal to the mean change in score divided by the SD of the change in score. ${ }^{9}$ An SRM value between 0.5 and 0.8 is considered moderate, and 0.8 or greater as good responsiveness. ${ }^{10}$ Median total vibration grades at $12,26,40$, and 52 weeks of follow up were compared with the median value at entry (Wilcoxon signed rank test). All analyses were performed using STATA 5.0 for Windows 95 (Stata Statistical software: Release 5.0. College Station, TX, USA; Stata Corporation, 1997). A $\mathrm{p}$ value $\leqslant 0.05$ was considered statistically significant.

\section{Results}

The stable group (54 female and 59 male patients; median age 56, range 14-84 years) had a median duration of symptoms of 5.1 years. These patients still had residual symptoms or signs (seven patients were bedbound, 14 required assistance or a device to walk short distances; the remaining patients were independent). The median total (arm+leg) vibration grade was 1 (range 0-8) at all three assessments. Good interobserver and intraobserver reliability values were shown for the RS tuning fork ("experienced" couple: interobserver weighted $\kappa$ values at the arms 0.67 ; legs 0.83 ; arms+legs 0.80; intraobserver values at the arms 0.98; legs 0.79; arms+legs 0.90; "variable" couples interobserver values at the arms 0.77 ; legs 0.82 ; arms+legs 0.86 ; intraobserver values at the arms 0.71 ; legs 0.85 ; arms+legs $0.84 ; \mathrm{p}<0.0001$ for all values).

Eight female and 12 male patients (median age 54.0, range 15-70 years) were examined longitudinally. At entry, the median total vibration grade was 4 (range $0-8$ ). Four of these patients $(20 \%)$ were initially bedbound, and nine $(45 \%)$ were unable to walk independently. 
A total of 185 visits were completed during a follow up period of 26-58 weeks. With the exception of one patient with GBS who only experienced mild symptoms, all patients received initial treatment with IVIg $(0.4 \mathrm{~g} / \mathrm{kg} /$ day for 5 consecutive days). All but one patient with CIDP showed good improvement on IVIg. The non-responder received a course of treatment with oral prednisone $(100 \mathrm{mg} /$ day $)$, for 4 consecutive weeks. This patient also improved with this therapy and prednisone was tapered down in 5 months to 30 mg every other day.

The patients with GBS did not show any deterioration. After initial improvement, all 12 IVIg responsive patients with CIDP showed deterioration in their clinical condition with increasing vibration sense abnormalities (compatible with higher vibration grades) at examination. Maintenance therapy with IVIg (1-2 days at $0.4 \mathrm{~g} / \mathrm{kg} /$ day at intervals of 3-21 weeks) was needed. Eventually, at 26 weeks of follow up, all but one patient with GBS (19/20=95\%) were independent in daily activities such as walking, dressing up, and eating with a knife and fork with decrements in symptoms and signs. At 1 year, the 13 patients who completed this period were also independent in daily activities with no or only minor symptoms or signs. Improvement in the longitudinal group resulted in a general reduction in total vibration grades (median values $2,0,0.5$, and 1 at weeks $12,26,40$, and 52, respectively) compared with the median entry value of 4 (Wilcoxon signed rank test $\mathrm{p}=0.01-0.0007$ ). Good SRM scores were obtained for the fork in these patients (SRM values 1.0, 1.3, 1.1, and 1.3 at weeks $12,26,40$, and 52 , respectively).

\section{Discussion}

In the current study, good interobserver and intraobserver reliability and high responsiveness were demonstrated for the Rydel-Seiffer (RS) graduated tuning fork in patients with immune mediated polyneuropathies. Hence, all psychometric requirements are accomplished for this fork by combining these results with literature findings. ${ }^{1211-13}$ Others have also shown acceptable reliability for this instrument. ${ }^{11-13}$ However, by contrast with the current study, these reliability values were reported only for distal examination in the limbs. ${ }^{11-13}$ Thivolet et al reported within test variations in the arms, but unfortunately vibration was only assessed at the thumbs. ${ }^{13}$ In the current study, more frequent use of the RS tuning fork did not show consist- ently higher reliability values, thus excluding a learning effect.

Vibration sense changes over time were adequately captured using the standardised response mean score (SRM), a statistical method of measuring responsiveness. ${ }^{9}$ However, the SRM scores obtained for the total vibration grades only showed responsiveness within one group of patients. Future studies are required to determine whether substantial discriminative responsiveness will be obtained for this fork when evaluating different groups of patients - for example, in a trial setting comparing a placebo versus a treatment group. ${ }^{14}$

In conclusion, good reliability values and responsiveness are provided for the RS graduated tuning fork in patients with immune mediated polyneuropathies. Based on these results combined with literature findings, it is suggested that this instrument should be incorporated in routine neurological examination, particularly for the assessment of vibration sense in patients with polyneuropathies.

This study was part of the Biomed project, number: BMH4-CT96 0324.

1 Martina ISJ, van Koningsveld R, Schmitz PIM, et al. Measuring vibration threshold with a graduated tuning fork in Neurosurg Psychiatry 1998;65:743-7.

2 Hobart JC, Lamping DL, Thompson AJ. Evaluating neurological outcome measures: the bare essentials. If Neurol Neurosurg Psychiatry 1996;60:127-30.

3 Hartung H-P, van der Meché FGA, Pollard JD. GuillainHartung H-P, van der Meche FGA, Pollard JD. Guillain-
Barré syndrome, CIDP and other chronic immunemediated neuropathies. Curr Opin Neurol 1998;11:497513.

4 Ad Hoc subcommittee of the American Academy of Neurology AIDS task force. Research criteria for diagnosis of chronic inflammatory demyelinating polyneuropathy (CIDP). Neurology 1991;41:617-18.

5 Asbury AK, Cornblath DR. Assessment of current diagnostic criteria for Guillain-Barré syndrome. Ann Neurol 1990; 27(suppl):S21-4.

6 Miescher GC, Steck AJ. Paraproteinaemic neuropathies. Baillieres Clin Neurol 1996;5:219-32.

7 Rydel A, Seiffer W. Untersuchungen über das vibrationsgefühl oder die sog. "Knochensensibilität" (Pallästhesie). Archiv für Psychiatrie und Nervenkrankenheiten 1903;37: A88-536.

8 Cohen J. Weighted kappa: nominal scale agreement with provision for scaled disagreement or part credit. Psychol provision for scaled

9 Liang MH, Fossel AH, Larson MG. Comparisons of five health status instruments for orthopedic evaluation. Med Care 1990;28:632-42.

10 Cohen J. Statistical power analysis for the behavioural sciences. New York, Academic Press, 1977.

11 Crausaz FM, Clavel S, Liniger C, et al. Additional factors associated with plantar ulcers in diabetic neuropathy. Diabet Med 1988;5:771-5.

12 Liniger C, Albeanu A, Bloise D, et al. The tuning fork revisited. Diabetes Care 1990;7:859-64.

13 Thivolet C, El Farkh J, Petiot A, et al. Measuring vibration sensation with graduated tuning fork. Diabetes Care 1990;13:1077-80.

14 Guyatt G, Walter S, Normal G. Measuring change over time: assessing the usefulness of evaluative instruments. $\mathcal{F}$ Chron Dis 1987;40:171-8. 